\title{
A Robust Video Information Hiding Communication Scheme Quantization-Based in CS Domain
}

\author{
Huimin Zhao $^{1 *}$, Jun Cai ${ }^{1}$, Li Zhu ${ }^{2}$ \\ ${ }^{1}$ School of Electronic and Information, Guangdong Polytechnic Normal University, Guangzhou 510665, \\ China. \\ 2 Industrial Training Center, Guangdong Polytechnic Normal University, Guangzhou 510665, China. \\ * Corresponding author. Tel.: 8602038256735; email: zhaohuimin@gdin.edu.cn \\ Manuscript submitted June 10, 2014; accepted October 8, 2014. \\ doi: 10.17706/ijcee.2014.v6.856
}

\begin{abstract}
In information hiding communication system, quantization-based video watermarking schemes are widely used. However, these approaches exist in some drawbacks due to the robustness while embedding the hidden information in spread spectrum (SS) solutions. On other hand, the additional hidden information (watermark) may often be that the distortion for the transform domain coefficients is large enough so as to make the retrieval of the hidden data impossible. In order to solve the problems, in this paper, we present a compressive sensing (CS) based watermarking communication scheme able to hide digital fingerprint images for privacy protection. We discuss the way of using CS on the host signal for watermarking communication purpose. The extensive experiments have shown that the proposed method can effectively obtain a video watermarking scheme in CS domain with interesting properties for protection the hidden fingerprint images, and achieve the best trade-off between robustness and statistical invisibility.
\end{abstract}

Key words: Information hiding, video watermarking, quantization, compressive sensing, fingerprint.

\section{Introduction}

Digital video is a very promising host signal that can carry a large amount of data (payload) and its potential for secret communications is largely unexplored. Now, after the host video, with embedded watermark data, has been subjected to compression, it may often be that the distortion for the transform domain coefficients is large enough so as to make the retrieval of the hidden data impossible, the distortion is mainly caused by perturbation of the coefficients [1], [2]. On the other hand, the allowable bit-rate of the video stream is determined via the bandwidth of the transmission medium. Thereby, it is a challenge to design a robust information hiding approach [2].

In this study, we propose to use the compressive sensing (CS) techniques to obtain a sparse representation of the host signal [3] before embedding the watermark. The great interest of such approach is that the compression-decompression process to be used are efficient can widely be used on real image extracted from video frame for our life applications. Moreover, it is well known that the transformed-domain watermarking techniques have been shown to be more robust and tamper-proof as compared to straightforward spatial watermarking [4], [5]. Other works like for example [6] or [7] has already proposed the use of CS approaches for watermarking purpose but to the best of our knowledge, no-one consider informed based watermarking techniques and neither propose to solve their lack of statistical transparency performance. 
Recently, application of the watermarking techniques have been introduced and shown to be promising for protecting fingerprint data and increasing the security level of fingerprint-based systems [8]. For example, watermarking of fingerprint images can be used to secure central databases from which fingerprint images are transmitted on request to intelligence agencies in order to use them for identification and classification purposes.

Considering the application of video watermarking for privacy protection in information hiding filed, the aim of our proposed scheme is to protect fingerprint data in the video host signal. With the secure watermarking principle [9], we evaluate the robustness using PSNR (Peak Signal-to-Noise Ratio) and $N C$ based on our previous work [10].

The rest of this paper is organized as follows. Section 2 describes the problems of the Scalar Costa Scheme (SCS). Section 3 introduces compressed sensing Costa Scheme (CS-SCS) proposed in this paper. Section 4 shows the experimental results for protecting fingerprint in video watermarking by the scheme. Finally, we give our conclusion in Section 5.

\section{Related Work for SCS Watermarking Communication Scheme}

For this work we consider the Costa's quantization based watermarking scheme [11] as the basis of our work. After brief reminder of general concepts of this scheme, we will present the major drawbacks of this scheme that we propose to enhance in this work.

\subsection{SCS Information Hiding Communication Scheme}

Let's consider a communication signal $X$ from a video document with the power $\sigma_{X}^{2}$. We wonder to embed a hiding message $m$ encoded with $X$ into the watermark signal $W$ (with the power $\sigma_{W}^{2}$ ) to be inserted in $X$ for producing the watermarked signal $S$ such as $S=X+W$. The watermarked signal is then transmitted through a disturbed communication channel. This disturbance is then modeled by a noise $Z$ of power $\sigma_{Z}^{2}$. At the receiver side, the noisy watermarked signal is obtained as the received signal $R$ such as $R=S+Z$. The costa's decoding process is then performed using $R$ to extract the embedding information and estimate the hidden message $\hat{m} n$. In Costa's approach, $X$ is supposed to be an I.I.D. (Independent Identically Distributed) signal, $W$ is supposed to be the weighted quantization error of the host signal $X$ such as $W=\alpha \cdot q$, where $q$ is the host signal quantization error using a scalar quantization with step $\Delta$, and $\alpha$ is the weighting coefficient. In case of a binary message (alphabet length $D=2$ ) with $d_{n}$ its elements, $q$ is denoted as

$$
q_{n}=Q_{\Delta}\left[X-\Delta\left(\frac{d_{n}}{D}\right)\right]-\left[X-\Delta\left(\frac{d_{n}}{D}\right)\right]
$$

At the receiver side $y$ is extracted during the decoding process as

$$
y_{n}=Q_{\Delta}(R)-(R)
$$

The message $\hat{m}$ with elements $d_{n}$ is then estimated by testing $\left|y_{n}\right| \leq \frac{\Delta}{2}$ which should be close to zero if $\hat{d}_{n}=0$ and close to $\pm \frac{\Delta}{2}$ if $\hat{d}_{n}=1$. To evaluate the statistical transparency of the embedded watermark, an analysis based on the Stein's Lemma is proposed here based on [12]. 


\subsection{Analysis of the SCS Scheme Based on the Stein's Lemma}

The aim of the statistical transparency, consist in reducing the ability for some attackers to easily distinguish a watermarked video to a non-watermarked one at transmission communication. If we consider the two hypothesis:

$$
\left\{\begin{array}{l}
H_{1} \text { : the signal is watermarked, its PDF is } p_{S} \\
H_{0} \text { : the signal is watermarked, its PDF is } p_{X}
\end{array} .\right.
$$

Here, PDF denotes probability density function of the signal. The two hypothesis mean the false alarm probability $P_{f a}$ can be maximized by considering the $P_{f a}=P_{r}\left(H_{1} \mid H_{0}\right)$, i.e. we want to maximize for the attacker to erroneously evaluate that a video is not watermarked. According to the Stein's Lemma with such hypothesis, we can obtain the Kullback-Leibler Divergence

$$
D\left(p_{X} \| p_{S}\right)=\int_{-\infty}^{+\infty} p_{X}(z) \ln \frac{p_{X}(z)}{P_{S}(z)} d_{z} \propto-\ln P_{f a}
$$

Therefore, maximizing the $P_{f a}$ corresponds to minimize the $D\left(p_{X} \| p_{S}\right)$ between the $p_{S}$ of the watermarked signal and the $p_{X}$ of the non watermarked signal. The perfect statistical transparency is very difficult to achieve $p_{S}=p_{X}$. Hence, C. Cachin [12] propose to feel free with this constraint by defining an $\varepsilon_{\text {secure }}$ system where $D\left(p_{X} \| p_{S}\right)<\varepsilon$. In this study, we will compute the KLD as described to evaluate the statistical transparency. Thus as the divergence will be small, higher will be the $P_{f a}$ and more confuse will be the attacker decision while trying to easily distinguish a watermarked to a non-watermarked signal.

\section{Compressed Sensing Scalar Costa Scheme (CS-SCS)}

\subsection{The CS-SCS Watermarking Communication Scheme}

The CS-SCS scheme proposed in this paper is described in Fig. 1. In Fig. 1, the host communication signal $X$ is compressed to obtain a sparse signal $\bar{X}$ in CS domain and the fingerprint watermark signal $W$ is computed according to the same procedure than the Costa's encoder process previously described but based on the obtained sparse signal $\bar{X}$. Thus the $W$ will be the weighted quantization error $(W=\alpha \cdot q)$ such as $q=Q_{\Delta}\left[\bar{X}-\Delta\left(\frac{d_{n}}{D}\right)\right]-\left[\bar{X}-\Delta\left(\frac{d_{n}}{D}\right)\right]$, and $\alpha$ is the Costa's robustness optimization parameter. It has to be noted that the quantization of the sparse signal will only be considered for the non-zero coefficients in CS domain.

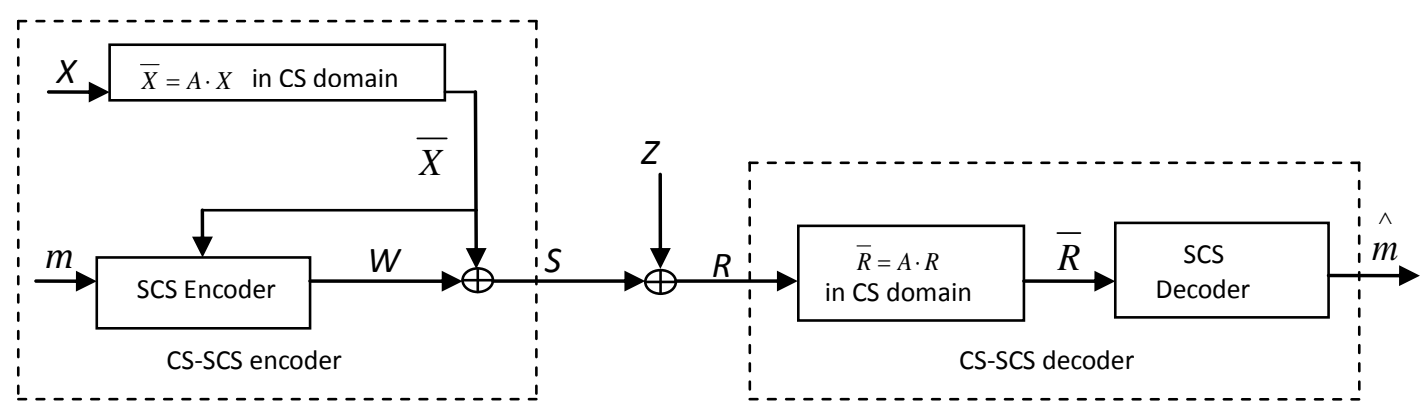

Fig. 1. The proposed CS-SCS watermarking communication scheme. 
This produced sparse watermarking information $W$ is added to $\bar{X}$ and then we obtain the watermarked sparse signal $S$ in CS-SCS encoder as shown in Fig. 1. The reverse CS operation is then done to obtain the watermarked signal in the original domain $S$. This signal is then transmitted through the noisy $Z$ channel $(Z$ denotes noise signal) and then sent to the receiver side.

At the receiver side, the noisy watermarked signal is received $R=S+Z$ and then compressed by using the same CS procedure applied during the embedding step. The sensing received signal $R$ is then used to extract the fingerprint watermark signal and estimate the hidden message $\hat{m}$ in CS-SCS decoder.

\subsection{Generation of the Watermarked Signal in CS domain}

The CS is a sampling paradigm that allows us to go beyond the Shannon limit by exploiting the sparsity structure of the signal in [13]. The CS allows us to capture and represent compressible signals at a rate significantly below the Nyquist rate [14]. The sampling step is very fast because it employs nonadaptive linear projections that preserve the structure of the signal. The signal is reconstructed from these projections by viewing the decoding step as a linear inverse problem that is cast as a sparsity regularized convex optimization problem [14]. The CS theory asserts that one can recover certain signals and images from far fewer measurements $\bar{X}$ or $\bar{R}$ than original samples $N$.

In Fig. 1 , assume $X$ is an input signal from original video stream, and $\bar{X}$ is a sparse signal in CS-SCS encoder, considered as a vector in a finite-dimensional subspace of $\mathfrak{R}^{n}$, then we can prove the $\bar{X}=\{\bar{x}[1], \ldots, \bar{x}[n]\}$ is strictly or exactly sparse if most of its entries are equal to zero, i.e. if its support $\Lambda(\bar{X})=\{1 \leq i \leq n, \bar{x}[i] \neq 0\}$ is of cardinality $k \ll n$. A $k$-sparse signal $\bar{X}$ is a signal for which exactly $k$ samples

\subsection{Extraction of the Watermark Signal in CS Domain}

In the Costa's philosophy, the watermark $W$ is homogenous to a scaled quantization error given by

$$
W=\alpha\left(Q_{\Delta}\left\{R D W T(X)-\Delta\left(\frac{d_{n}}{D}\right)\right\}-\left\{R D W T(X)-\Delta\left(\frac{d_{n}}{D}\right)\right\}\right)
$$

In Fig. 1, the watermark is embedded in the sparse signal $\bar{X}=R D W T(X)$ according to the principle of $S=\bar{X}+W$. Using the property that the gradient for natural fingerprint image generally follows a heavy-tailed distribution, we consider the Total Variation (TV) optimization problem such as min- TV(S) subject to $Z=I R D W T(S)$ where $I R D W T(S)$ stands for inverse RDWT in CS domain and

$$
T V(S)=\sum_{i j} \sqrt{D_{h ; i j}^{2} S+D_{v ; i j}^{2} S}
$$

Here

$$
\begin{aligned}
& D_{h ; i j} S=\left\{\begin{array}{llc}
S_{i+1, j}-S_{i j} & \text { if } & i<n \\
0 & \text { if } & i=n
\end{array}\right. \\
& D_{v ; i j} S=\left\{\begin{array}{llc}
S_{i, j+1}-S_{i j} & \text { if } & j<n \\
0 & \text { if } & j=n
\end{array}\right.
\end{aligned}
$$

We can finally obtain a watermarked image $S$ which is then reconstructed with a satisfying PSNR computed between the watermarked image $S$ and the original one $X(P S N R \geq 48.56 d B)$. As is has been proved that $P S N R$ and mean squared error (MSE) approaches, are not enough consistent with human eye 
perception to give a good perceptual transparency evaluation [15]. The Structural Similarity coefficient (SSIM) is computed between $X$ and $S$, and its value $(S S I M \geq 0.97)$ confirm the good perceptual transparency performance for the watermarked signal reconstruction in [16].

\section{Experimental Results}

To test and verify the performance of the proposed scheme for use to protect fingerprint images in which the CS procedure was applied to every frame of the video document. The experimental results are compared with SCS's method in [11] and A. Abdulfetah's method in [4] to perform various attacks, including MPEG compression, noise contamination.

\subsection{Experiment Objects}

In our experiment, the watermarking message $m$ is generated by measurements values of sensing matrix $\Phi$ for a gray level fingerprint image with the size $160 \times 160$. For host video signal $X$, experimental frames extracted from two real video documents, Basketball and Scene, for demonstration. The each frame size of each video is $720 \times 480$, and every video consists of 300 frames.

\subsection{Experiment of Statistical Transparency}

For our proposed scheme, by computing the KLD for two watermarked video signals with (3), we are able to prove that along with the document-to-watermark ratio (DWR) its value obtained with our proposed CS-SCS's scheme is widely lower than the ones obtained for the SCS's scheme and A.Abdulfetah's scheme as shown in Fig. 2 (a) and (b), respectively. This means that the CS-SCS's scheme can be said $\varepsilon_{\text {secure }}$ with $\varepsilon=6 \times 10^{-3}$.With the good statistical transparency performance obtained for this proposed CS-SCS's scheme we have to check the all the other watermarking performance.

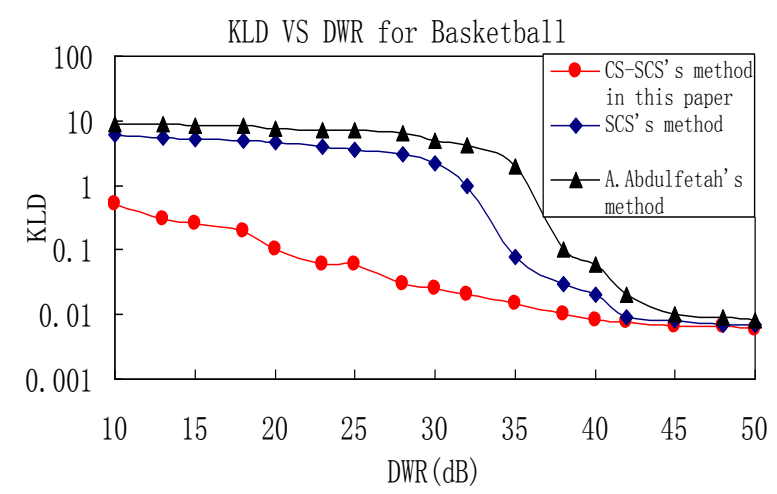

(a)

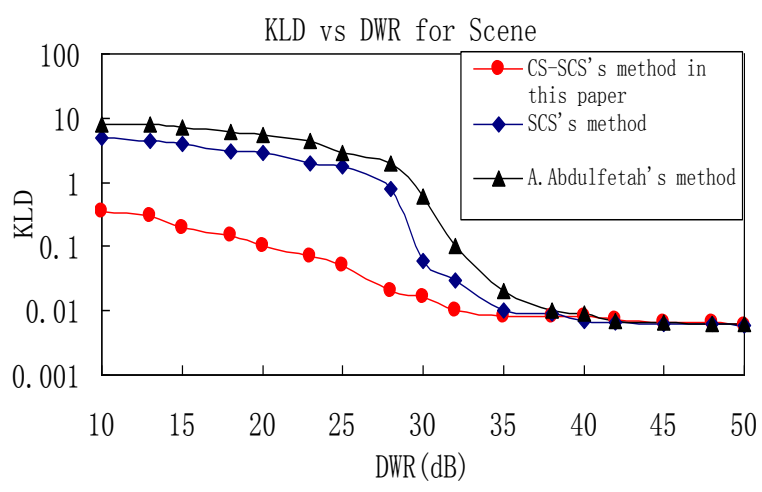

(b)

Fig. 2. KLD comparison with the proposed CS-SCS's scheme and other ones.

(a) Basketball video document; (b) Scene video document.

\subsection{Experiment of Compress Process}

We use MPEG-2 compression to evaluate the robustness of the proposed scheme. Figures.3 show the results after those of compressions under the different bit rates compared with our method, CS-SCS's method, and A. Abdulfetah's method. Obviously, the higher the NC value is, the better the robustness of the proposed ISCS scheme is. In other words, after embedded the fingerprint watermark signal, the hidden fingerprint data can almost be extracted in receiver even though the bit rate is as low as $0.5 \mathrm{Mbps}$ by using CS-SCS's method. The results show also that our proposed CS-SCS's method can efficiently resist compression attacks of MPEG-2 under transmission systems of the different bit rates. 


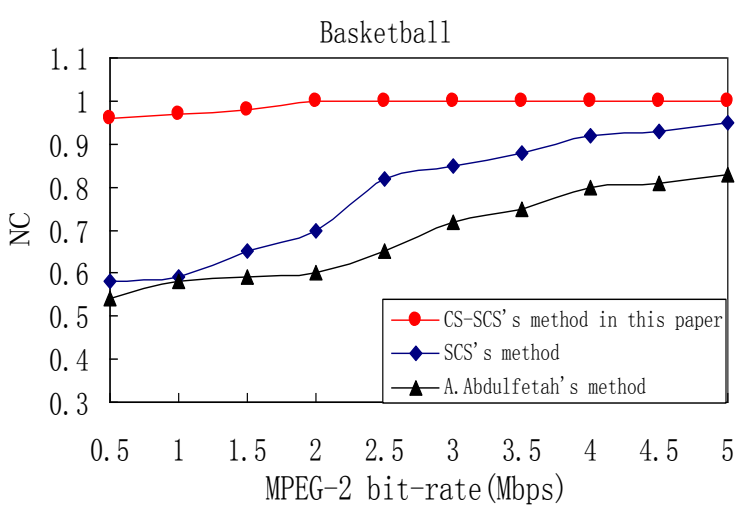

(a)

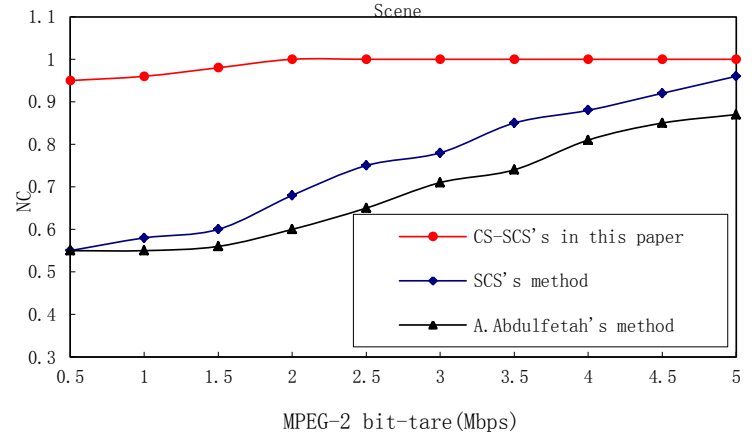

(b)

Fig. 3. Comparison result under the different MPEG-2 bit rates.

(a) Basketball video; (b) Scene video.

\subsection{Experiment of AWGN Channel Conditions}

In order to study deeply the robustness of the CS-SCS scheme for communication system, we consider also channel additive white Gaussian noise attacks. Fig. 4-Fig. 5 present the experimental results of the fingerprint image recovered with retracted watermark data by three methods after AWGN attacks in CS-SCS decoder. From these results, we can understand no matter what the attacks are, the NC values of the fingerprint image recovered from our proposed CS-SCS scheme can still exceed 0.98, and the image can reconstruct with higher quality than the methods of SCS and A.Abdulfetah.

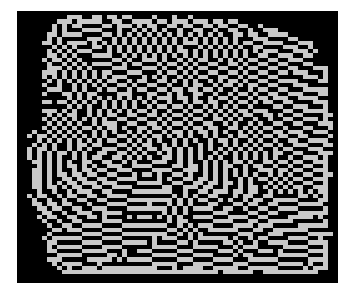

(a)

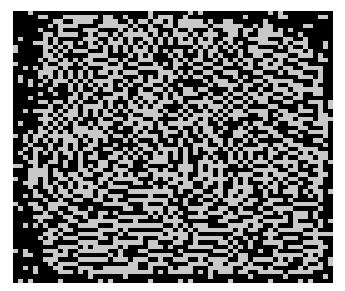

(b)

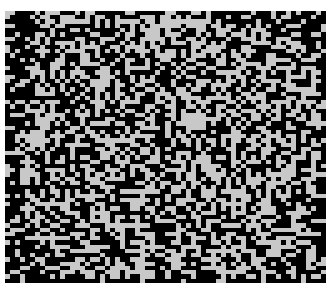

(c)

Fig. 4. Comparison result against AWGN attack for basketball video.

(a) Our proposed method, $\mathrm{NC}=0.98$. (b) SCS's method, $\mathrm{NC}=0.71$. (c) A. Abdulfetah's method, $\mathrm{NC}=0.59$.

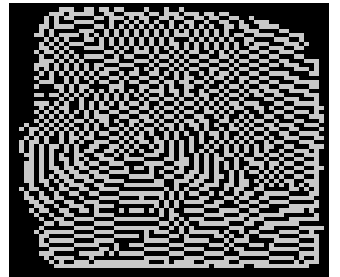

(a)

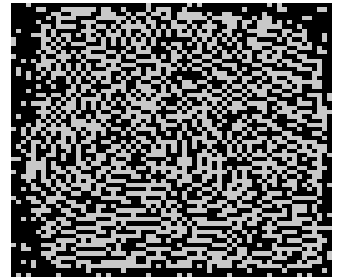

(b)

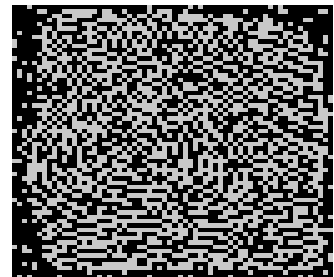

(c)

Fig. 5. Comparison result against AWGN attack for scene video.

(a) Our proposed method, $\mathrm{NC}=0.99$. (b) SCS's method, $\mathrm{NC}=0.75$; (c) A. Abdulfetah's method, $\mathrm{NC}=0.68$.

\section{Conclusion}

In this paper, we have proposed a robust CS-SCS video watermarking communication scheme quantized-based associated with the CS theory for protecting fingerprint images. The extensive experiments have shown that we are able to reach better robust performances compared to popular Scalar Costa Scheme and general video watermarking scheme using visual models in DWT domain. 


\section{Acknowledgment}

The authors wish to thank Prof. Jiwu Huang and Doctor Yanmei Fang of Sun Yat-sen University for insightful comment. This work was supported in part by the National Natural Science Foundation of China under Grant No. 61272381, in part by Science and Technology Project of Education Department of Guangdong Province of China under Grant No.2013KJCX0118, and in part by Science and Technology Project of Guangzhou City of China under Grant No.2014J4100078.

\section{References}

[1] Biswas, S., Das, R., \& Petriu, M. (2005). An adaptive compressed MPEG-2 video watermarking scheme. IEEE Transactions on Instrumentation and Measurement, 54(5), 1853-1861.

[2] Barni, M., Bartolini, F., \& Checcacci, N. (2005). Watermarking of MPEG-4 video objects. IEEE Transactions on Mutimedia, 7(1), 23-32.

[3] Starck, J. L., Murtagh, F., \& Fadili, J. M. (2010). Sparse Image and Signal Processing: Wavelets, Curvelets, Morphological Diversity. Cambridge University Press.

[4] Abdulfetah, A., Sun, X., \& Yang, H. (2010). Robust adaptive video watermarking scheme using visual models in DWT domain. Information Technology Journal, 9(7), 1409-1414.

[5] Chetan, K., \& Raghavendra, K. (2010). DWT based blind digital video watermarking scheme for video authentication. International Journal of Computer Applications, 4(10), 19-26.

[6] Lu, W., Varna, A. L., \& Wu, M. (2010). Security analysis for privacy preserving search for multimedia. Proceedings of IEEE 17th Inter. Conf. on Image Processing.

[7] Wang, Q., Zeng, W. J., \& Tian, J. (2013). Integrated secure watermark detection and privacy preserving storage in the compressive sensing domain. IEEE International Workshop on Information Forensics and Security (pp. 67-72).

[8] Zebbiche, K., Ghouti, L., Khelifi, F., \& Bouridane, A. (2006). Protecting fingerprint data using watermarking. Proceedings of the 1st NASA/ESA Conference on Adaptive Hardware and Systems (pp. 451-456), Turkey.

[9] Bianchi, T., \& Piva, A. (2013). Secure watermarking for multimedia content protection: A review of its benefits and open issues. IEEE Signal Processing Magazine, 30(2), 87-96.

[10] Zhao, H. M., Lai, J. H., Cai, J., \& Chen, X. L. (2013). A video watermarking algorithm for intraframe tampering detection based compressed sensing. Acta Electronica Sinica, 41(6), 1153-1158.

[11] Eggers, J. J., Bauml, R., Tzchoppe, R., \& Girod, B. (2003). Scalar costa scheme for information embedding. IEEE Trans. on Signal Processing, 51(4), 1003-1019.

[12] Cachin, C. (1998). An information-theoretic model for steganography. Lecture Notes in Computer Science, 1525, 306-318.

[13] Candes, E. J., Wakin, M. B., \& Romberg, J. (2008). An introduction to compressive sampling. IEEE Signal Processing Magazine, 25(2), 21-30.

[14] Candes, E. J., \& Romberg, J. (2009). $l_{1}$-Magic: Recovery of sparse signals via convex programming. In Svetlana Avramov-Zamurovic (Eds.) Compressive Sensing Tutorial, part 2.

[15] Fowler, J. E., Mun, S. W., \& Tramel, E. W. (2011). Multiscale block compressed sensing with smoothed projected Landweber reconstruction. Proceedings of $19^{\text {th }}$ European Signal Processing Conference (pp. 564-568), Barcelona.

[16] Wang, Z., Bovik, A. C., Sheikh, H. R., \& Simoncelli, E. P. (2004). Image quality assessment: From error visibility to structural similarity. IEEE Transactions on Image Processing, 13(4), 1-14. 


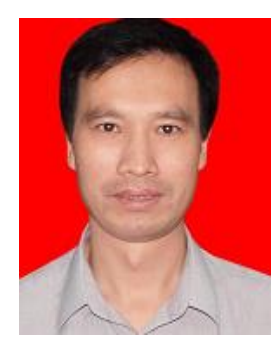

Huimin Zhao was born in Shanxi, China, in 1966. He received the B.Sc. and M.Sc. degrees in signal processing in 1992 and 1997 from Northwestern Polytechnical University, Xian, China, respectively. He received the Ph.D. degree in electrical engineering from the Sun Yat-sen University in 2001. At present, he is a professor of the Guangdong Polytechnic Normal University. His research interests include image, video and information security technology.

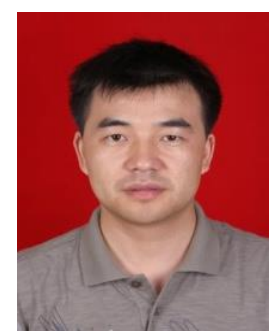

Cai Jun received the B.S degree from Hunan Normal University, Changsha, China, the M.S degree from Jinan University, Guangzhou, China, and the Ph.D. degree from Sun Yat-Sen University, China in 2003, 2006 and 2012, respectively. He is currently an instructor with the School of Electronic and Information, GuangDong Polytechnic Normal University, Guangzhou, China. Complex network, traffic modeling and anomaly detection have been of particular interest over recent years.

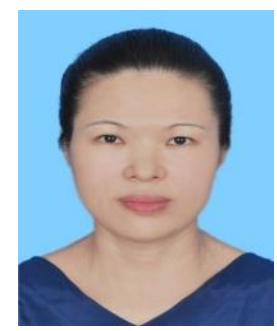

Li Zhu was born in Guangxi, China, in 1969. She received the B.S. degree in pressure processing from Northwestern Polytechnical University, China, in 1991, and the M.S. degree in engineering computer technology, Guangdong University of Technology, China, 2007. At present, she is a senior engineer of the Guangdong Polytechnic Normal University. Her research interests include multimedia processing, computer vision and information security technology. 